\title{
Designing Omni-Directional Mobile Robot with Mecanum Wheel
}

\author{
${ }^{1}$ Jefri Efendi Mohd Salih, ${ }^{1}$ Mohamed Rizon, ${ }^{1}$ Sazali Yaacob, \\ ${ }^{1}$ Abdul Hamid Adom and ${ }^{2}$ Mohd Rozailan Mamat \\ ${ }^{1}$ School of Mechatronics Engineering, Kolej Universiti Kejuruteraan Utara Malaysia \\ 01000 Kangar, Perlis, Malaysia \\ ${ }^{2}$ Terengganu Advanced Technical Institute, Jalan Panchor, Teluk Kalong \\ 24000 Kemaman, Terengganu, Malaysia
}

\begin{abstract}
This study presents the processes undertaken in the design and development of an intelligent omni-directional mobile robot using four custom-made mecanum wheels. The mecanum wheel developed consists of nine rollers made from delrin. All mecanum wheels are independently powered using four units of precisian gear DC motors and the wheel/motor assemblies were mounted directly to the robot chassis made using an aluminum frame. A four channel high power H-bridge using 2 units of LMD 18200 motor drivers IC circuit was design, built and interfaced to a BasicStamp (BS2) microcontroller board. Basic mobility algorithm using BasicStamp software was developed to test the basic mobility capabilities and test the qualitative view of the system's mobility performance. An experiment was set-up to analyze the motion characteristic of the mobile robot motion in Y-axis, $\mathrm{X}$ axis and rotary motion. Data from the experiment will be used for mathematically model for mobile robot platform and speed controller modeling and design. Mecanum wheel mobile robot provides a convenient platform for further development in the mobile platform. The combination of mechanical design on the wheel and chassis, motion control and multiple input/output sensors allow the exploration of large number of control algorithm and software to be implemented to the robot for practical applications.
\end{abstract}

Key words: Mecanum wheel, mobile robot, microcontroller, motion control

\section{INTRODUCTION}

This project utilized the Mecanum wheel design pioneered in 1973 by Mecanum AB's Bengt Ilon. Mecanum wheel is based on the principle of a central wheel with a number of rollers placed at an angle around the periphery of the wheel. The angled peripheral roller translates a portion of the force in the rotational direction of the wheel to force normal to the wheel directional. Depending on each individual wheel direction and speed, the resulting combination of all these forces produces a total force vector in any desired direction thus allowing the platform to move freely in direction of resulting force vector, without changing the direction of the wheel. Figure 1 shows a traditional Mecanum wheel design by Ilon with the peripheral roller with $45^{\circ}$ degree slope held in place from the outside.

Using four of mecanum wheels provides omnidirectional movement for a vehicle without needing a conventional steering system Slipping is a common problem in the mecanum wheel as it has only one roller with a single point of ground contact at any one time.

Due to the dynamics of the mecanum wheel, it can create force vectors in both the $\mathrm{x}$ and $\mathrm{y}$-direction while

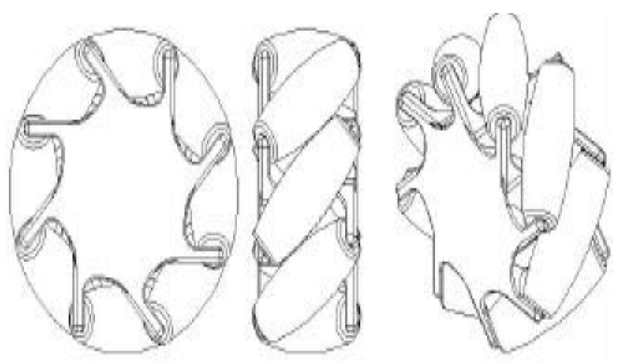

Fig. 1: Mecanum wheel based on Ilon's concept, Source from ${ }^{[1]}$

only being driven in the y-direction. Positioning four mecanum wheels, one at each corner of the chassis (two mirrored pairs), allows net forces to be formed in the $\mathrm{x}$, $\mathrm{y}$ and rotational direction (Fig. 2).

A difficulty with this strategy is that there are four variables to control three degrees-of-freedom. In this case the system is said to be over determined and it is possible to create conflicts in the actuation. As a result of the constraints associated with the mecanum wheel some form of controller is required to produce satisfactory motion. 


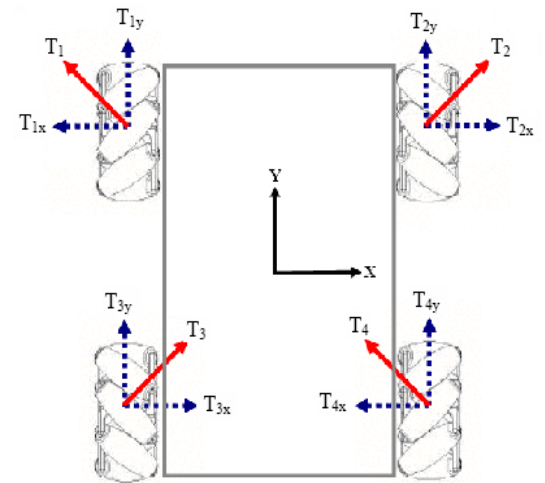

Fig. 2: Force vectors created by Mecanum wheel

\section{MATERIALS AND METHODS}

As this was a complete Mechatronic project incorporating mechanical, electronic and software development, the different areas were developed synergistically thus allowing interactions between the disciplines to be viewed and managed. It also meant that all three core disciplines needed to be developed to a certain stage before any one area could be further worked on. Although it was physically possible to use other means to develop the core areas independently, a synergistic approach tends to be more efficient. Even though this parallel design approach was used, the areas of development shall be discussed in sections assuming that other sections have already been completed to a certain level and are referenced where necessary.

Developments and implementation: The development for this project can be divided into the major process, the mechanical design for mecanum wheel and mobile robot chassis, electronics design for 4 channel motor driver and interfacing with BasicStamp microcontroller board and software development for motion control.

Mechanical design: The mecanum wheel been develop consist of nine roller with diameter of $110 \mathrm{~mm}$. Each roller diameter is $20 \mathrm{~mm}$ at the center and $16 \mathrm{~mm}$ at each end. All rollers are made by engineering plastic call delrin. The roller was hold by roller holder made by stainless steel and the center hub was made aluminum. Design structure of the mecanum wheel shown in Fig. 3. All mecanum wheels are independently powered using four units of precisian gear DC motor and the wheel/motor assemblies were mounted directly to the robot chassis.

Typical mecanum wheel mobile robot platforms are square or rectangular, attach with wheel with $+45^{\circ}$ roller and wheel with $-45^{\circ}$ roller on each side. The omni-directional capabilities of the platform depend on each wheel contact firmly with the surface and some of the mecanum wheel mobile robots are equipped with suspension system. For simplicity our mecanum wheel and motor assembly are mounted directly on the platform chassis made from aluminum frame and metal plate.
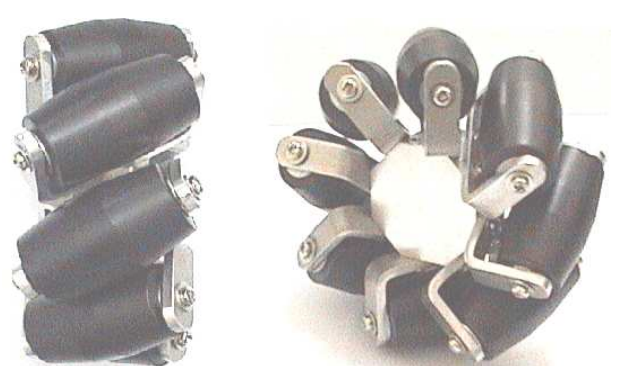

Fig. 3: Design structure of the mecanum wheel

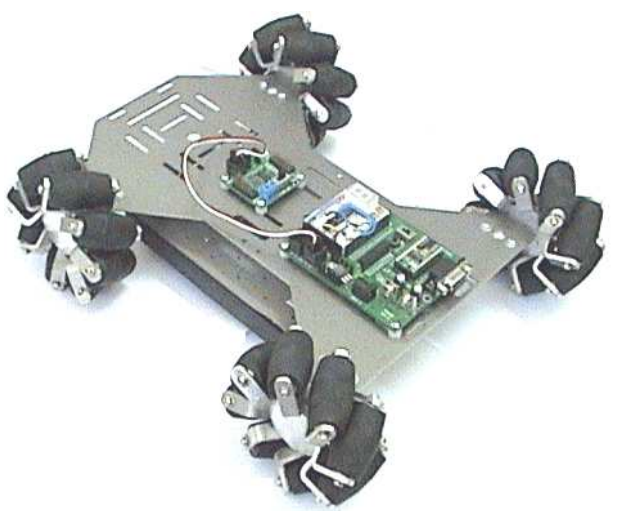

Fig. 4: Design structure of mecanum wheel mobile robot

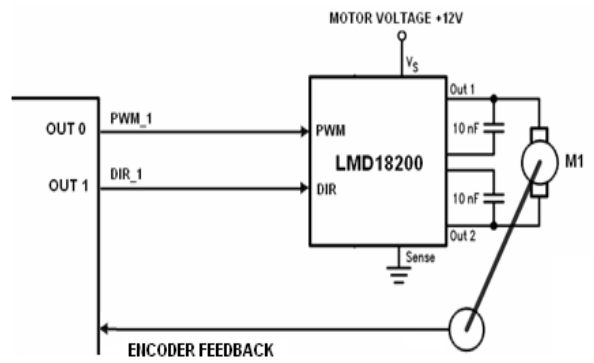

Fig. 5: Single motor driver circuit

The size of mobile robot approximately $20 \mathrm{~cm}$ (width) and $30 \mathrm{~cm}$ (length). Figure 4 show the design structure of the mobile robot.

Electronic design: Four channel bi-directional motor driver been design to drive all four mecanum wheels. The specifications developed for the necessary driver board were:

* The circuit should be compatible with a single logic-level PWM input signal for speed control of each wheel and a single logic-level input line for the direction of motor rotation for each wheel.

* The circuit should be able to operate with a high PWM carrier frequency from the microcontroller $(20 \mathrm{MHz})$ to provide inaudible operation.

* The circuit would require four independent $\mathrm{H}$ Bridge drivers for bi-directional motion. 
* Each H-Bridge driver circuit must be capable of providing suitable continuous current at $12 \mathrm{~V} \mathrm{DC}$.

The prototype motor driver was develop using 2 units of LMD18200 IC manufactured by National Semiconductor that capable of 3 amps continuous current at up to 55V DC and also had extra integrated features including current sensing and thermal overload shutdown ${ }^{[2]}$. Figure 5 show the single motor driver circuit.

The DC motors used in this platform have built-in 40:1 gear reduction and speed at 65 RPM at 12VDC. The optical encoders provided velocity information on each wheel to the micro-controller. A four channel high power H-bridge driver board was interfaced to a BasicStamp (BS2) micro-controller board. The overall system hardware architecture (Fig. 6) shows the connections between hardware components of the mobile robot platforms.

Microcontroller: In order to give the existing robot any intelligent functionality some form of on-board processor was essential. Microcontrollers are ideally suited for such an application as they are compact, have many built-in hardware features such as timers and UARTS, have a significant number of digital I/O lines and have low power requirements. The essential microcontroller specification for this project was its ability to generate four independent PWM signals. Other general requirements were; high speed operation to ensure environmental data could be processed at realtime. BASIC Stamp micro-controllers have been chosen this project for well-known for their ease of use, comfortable programming language and easy debugging using a PC. Figure 7 show the BasicStamp (BS2) microcontroller board.

Table 1 and 2 show the relationship between output data of the micro-controller for speed (PWM) and bidirectional (DIR) control for all four DC motors and the omni-directional motion of the mecanum wheel mobile robot.

Experiment setup: With the use of the prototype motor driver board and test software to programming the microcontroller output as list in Table 2 and 3, the basic mobility control was gained via programming the basic motion software to the microcontroller. This setup allowed the following motions as well as the increase and decrease of speed.

* Forward - all four wheels forward in

* Backward - all four wheels move backward at the same speed

* $\quad$ Right slide - wheel 1 and 4 forward, wheel 2 and 3 backward

* $\quad$ Left slide - wheel 2 and 3 forward, wheel 1 and 4 backward.

* Clockwise - wheel 1 and 3 forward, wheel 2 and 3 backward

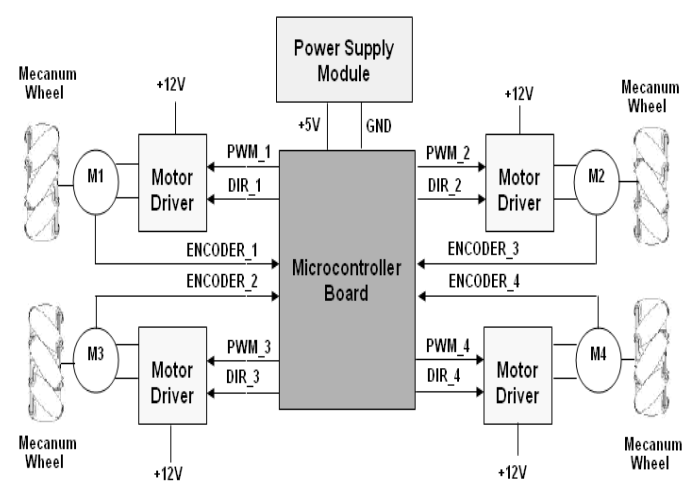

Fig. 6: System hardware architecture

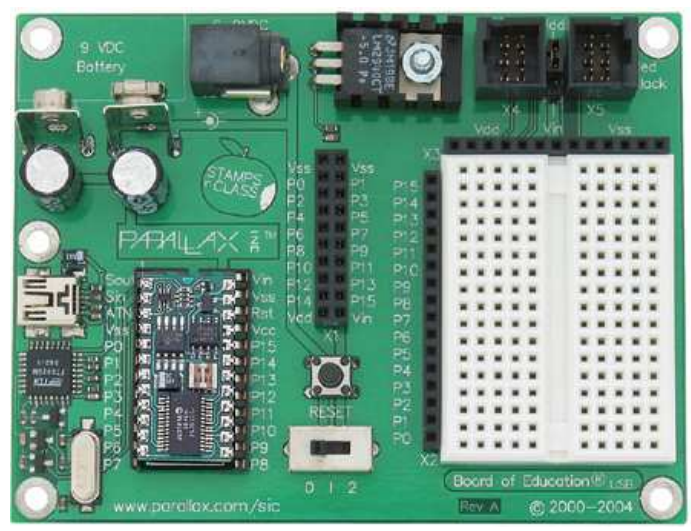

Fig. 7: BasicStamp microcontroller board

* Counter-Clockwise - wheel 1 and 3 backward, wheel 2 and 3 forward

The following list in Table 2, show the basic motion of mecanum wheel mobile robot with their corresponding wheel direction. By varying the individual motor/wheel speed we can achieve driving direction along any vector in $\mathrm{X}-\mathrm{Y}$ axis. The actuation required for these movements can be seen in Fig. 8 .

\section{RESULTS AND DISCUSSION}

The testing gave a qualitative view of the system's mobility performance. The forward and reverse motion as acceptable but did not utilize any function of the mecanum wheels. Likewise with rotational motion, the system performed as would be expected of a standard differential drive platform. The translational motion in $\mathrm{X}$ - axis, however, was not acceptable as the platform would tend to wander in the y-direction when attempting to traverse sideways. Investigation showed that the motor systems were working adequately but the mecanum wheels themselves and mobile robot chasis needed some minor modification.

A qualitative view of the platform's basic mobility for translational movement showed an increase in performance but at this stage some form of quantitative 


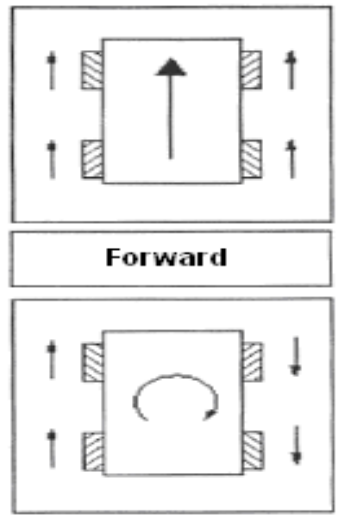

Rotate clockwise

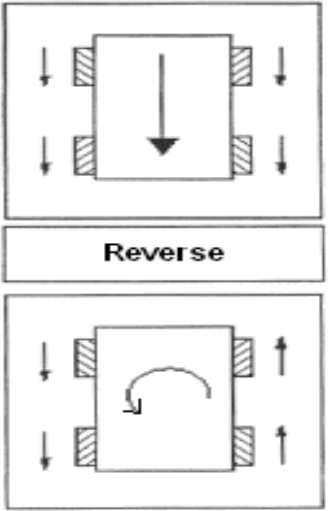

Rotate counter

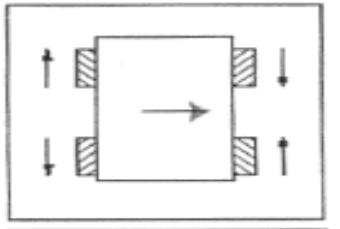

Right slide

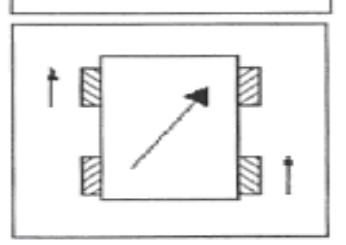

Turn Right

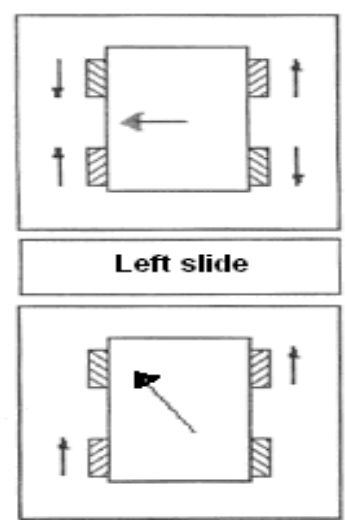

Turn Left

Fig. 8: Required actuation for general movement, Source from ${ }^{[3]}$

Table 1: Switch control on microcontroller output

\begin{tabular}{|c|c|c|c|c|c|c|c|c|}
\hline \multicolumn{3}{|c|}{ Output Pin } & \multicolumn{6}{|l|}{ Data } \\
\hline \multicolumn{3}{|l|}{0} & \multicolumn{6}{|c|}{ PWM input for M1 (High = 100\% PWM) } \\
\hline \multicolumn{3}{|l|}{1} & \multicolumn{6}{|c|}{ Direction input for M1 (High= Clockwise, Low=Counter Clock-Wise) } \\
\hline \multicolumn{3}{|l|}{2} & \multicolumn{6}{|c|}{ PWM input for M2 (High = $100 \%$ PWM $)$} \\
\hline \multicolumn{3}{|l|}{3} & \multicolumn{6}{|c|}{ Direction input for M2 (High= Clockwise, Low=Counter Clock-Wise $)$} \\
\hline \multicolumn{3}{|l|}{4} & \multicolumn{6}{|c|}{ PWM input for M3 (High = $100 \%$ PWM) } \\
\hline \multicolumn{3}{|l|}{5} & \multicolumn{6}{|c|}{ Direction input for M3 (High= Clockwise, Low=Counter Clock-Wise) } \\
\hline \multicolumn{3}{|l|}{6} & \multicolumn{6}{|c|}{ PWM input for M4 (High = $100 \%$ PWM) } \\
\hline \multicolumn{3}{|l|}{7} & \multicolumn{6}{|c|}{ Direction input for M4 (High= Clockwise, Low=Counter Clock-Wise) } \\
\hline \multicolumn{2}{|l|}{$\bar{M} 1$} & M 2 & \multicolumn{3}{|c|}{ M 3} & \multicolumn{2}{|l|}{ M 4} & \multirow[b]{2}{*}{ Basic Motion } \\
\hline PWM_1 & DIR_1 & PWM_2 & DIR_2 & PWM_3 & DIR_3 & PWM_4 & DIR_4 & \\
\hline High & High & High & High & High & High & High & High & forward \\
\hline High & Low & High & Low & High & Low & High & Low & backward \\
\hline High & Low & High & High & High & High & High & Low & right slide \\
\hline High & High & High & Low & High & Low & High & High & left slide \\
\hline High & High & High & Low & High & High & High & Low & Turning clockwise \\
\hline High & Low & High & High & High & Low & High & High & Turning counter-clockwise \\
\hline
\end{tabular}

analysis was required to review further minor modifications to the system especially on the mecanum wheel design and close-loop speed control on each motor. Data from the experiment will be used for mathematically model for mobile robot platform and speed controller modelling and design.

The accuracy of direction and movement of the mobile robot depend much on slip rate of the wheel on floor' various conditions. As are result, the real position and orientation of the mobile robot deviate from the original planned course or path. The other parameters that influence the accuracy of movement of mecanum wheel mobile robot are surface contact and traction. Because of slippage problem, motion analysis for mecanum wheel type vehicle is complicate. Visual dead reckoning for motion control and odometry are the method to reduce the motion error.

Close loop control using model base method or classical cascade PID for speed controller of mobile robot must be integrated into control algorithm to maintain set-point speeds in both the $\mathrm{x}$ and $\mathrm{y}$-directions.
The combination of these two speeds gives the actual speed and more importantly direction of the robot.

Future work: Mecanum wheel mobile robot provided a convenient platform for continued development. The combination of mechanical design on the wheel and chassis, motion control and multiple input/output sensors allow the exploration of large number of control algorithm and software to be implemented to the robot for practical application.

We are currently developing a tele-operated mecanum wheel mobile robot using RF module for data communication between robot and host computer. The mobile robot will implement closed-loop wheel speed control using fuzzy logic algorithm for robot omnidirectional kinematics and motion control.

The mecanum wheel also will be used as our platform for Autonomous Guided Vehicle (AGV) in Flexible Manufacturing System (FMS) at Robotic and Automation laboratory in KUKUM. The used of mecanum wheel AGV with the lifting mechanism on 
the AGV platform will be part of FMS system and optional with the current AGV that used differential drive and gantry robot at the ASRS station

\section{CONCLUSION}

This paper presents an overview over the primary design stage of omni-directional mobile robot using mecanum wheel. The strength of this wheel is the enhanced maneuverability of the mobile robot that needs extreme maneuverability in congested environment. Mecanum wheel mobile robot research addresses many problems in robotics such as sensor integration, real-world modelling, actuator and sensor control, path planning and navigation, task-level planning and execution and the control of the robotic system as a whole. Moreover, building mecanum wheels mobile robot provides a stringent test bed for new concepts and approaches in both mechanical design for mecanum wheel and overall mobile robot chassis and also the design for electronic hardware and software.
This design and development of an omnidirectional platform, using mechatronics system and mecanum wheel to implement intelligent behaviours and maneuvers, with the help of a microcontroller interfaced with sensors.

\section{REFERENCES}

1. Fiegel, O., A. Badve and G. Bright, et al., 2002. Improved mecanum wheel design for omnidirectional robots. Proc. Australasian Conf. Robotics and Automation, 27-29 Nov., pp: 117121.

2. Regan, T., 1999. A DMOS 3A,55V H-Bridge: LMD18200. National Semiconductor Application Note 868.

3. Phillips, J.G., 2000. Mechatronic design and construction of an intelligent mobile robot for educational purposes. Master of Technology Thesis, Massey University, Palmerston North, New Zealand, pp: 150. 